\title{
Low Dose Magnesium Sulphate Regime for Eclampsia and Imminent Eclampsia
}

\author{
Manisha M. Laddad, Anjali Patil, P. A. Havale, S.S. Vhawal, Bahulekar Ashitosh
}

Section: Healthcare

Sci. Journal Impact

Factor: $6.1(2018)$

ICV: 90.90 (2018)

Department of OB/GYN, Krishna Institute of Medical Sciences Deemed To Be University, Karad, India.

Copyright@IJCRR

\section{ABSTRACT}

Introduction: In India especially in rural areas, the majority of eclampsia cases report to hospital in an emergency. They are unregistered with inadequate antenatal care and travel a long distance before reaching the hospital as the rural health centres are not sufficiently equipped to manage this condition.

Aim: To determine the efficacy of low dose magnesium sulphate in eclampsia and imminent eclampsia. To measure serum levels of magnesium with this regime. To correlate serum magnesium levels and efficacy. To identify complications of magnesium sulphate therapy in the mother and the baby.

Methods: 120 cases of preeclampsia and eclampsia were allotted by simple random sampling technique to two groups i.e. low dose regime group and Pritchard regime group. It was observed that the majority of the cases were unbooked (90\%) and were in the age group of $21-25$ years $(76.6 \%)$.

Results: Study observed that eclampsia was primarily a disease of primigravida (61\%). Most women in the study group had a bodyweight of fewer than $50 \mathrm{kgs}$ (74\%). The average total dose of magnesium sulphate required was 20 grams i.e. $54.5 \%$ less than that required in standard Pritchard regime. Majority of the cases (90\%) responded to the low dose regime with the recurrence rate being $(10 \%)$ as compared to Pritchard regime group being $(91.6 \%)$ and recurrence being $8.33 \%$. The success rate for seizure prophylaxis in imminent eclampsia being $100 \%$ in both the groups. The average serum magnesium levels low dose regime in sample 1 was $3.36 \pm 0.76$ and $3.30 \pm 0.69$ for sample 2 . The therapeutic serum magnesium levels for the present low dose regime being 2.61 to $4.05 \mathrm{mg} / \mathrm{dl}$. The cost of therapy while doing so was reduced to less than half with low dose regime than that of Pritchard regime. The perinatal mortality was $30 \%$ with low dose regime.

Conclusion: Low dose magnesium sulphate regime was effective in seizure prophylaxis in imminent eclampsia patients and as effective as Pritchard regime in eclampsia patients. The dose required for control of convulsions was less than half used in Pritchard regime and even cost of therapy was half than Pritchard regime..

Key Words: Low Dose, Magnesium Sulphate, Eclampsia, Imminent Eclampsia, Seizure prophylaxis

\section{INTRODUCTION}

According to Bernhart et al. ${ }^{1}$ eclampsia has been known to mankind since ancient times. There is a mention about eclampsia in ancient Egyptian, Chinese, Greek and Indian literature. It has been an object of intense research and the subject of many theories, creating new diagnostic and therapeutic complications adding many unusual medical challenges. Hypertensive disorders of the pregnant account for the third-highest cause of maternal deaths and a high perinatal mortality. ${ }^{2}$
In India, the incidence of eclampsia ranges from 1 in 30 to 1 in 500 pregnancies. Thus eclampsia remains one of the most common causes of maternal and perinatal mortality and morbidity.

The first principle of management of eclampsia is the control of convulsion. Since the exact aetiology of the disease is not known, various drug modalities and regimen have been advocated. By far, amongst the various anticonvulsants used, magnesium sulphate gained popularity and is the most widely used anticonvulsant. Thus being the most widely used anticonvulsant, even large trials have been performed to study

\section{Corresponding Author:}

Dr. Anjali Patil, Department of ob/gyn, Krishna Institute of Medical Sciences Deemed To Be University, Karad, India. Email: hodobgyn@kimsuniversity.in

ISSN: 2231-2196 (Print) ISSN: $0975-5241$ (Online)

Received: 20.06.2020

Revised: 12.07.2020

Accepted: 16.08 .2020 Published: 08.09 .2020 
the clinical efficacy of magnesium sulphate therapy. One was the multinational eclampsia trial collaborative group $(1995)^{3}$, a study funded by World Health Organisation and an even larger study more than 10000 women with preeclampsia were randomly given magnesium sulphate i.e. the MAGPIE Trial Collaborative Group (2002) $)^{4}$. Following the publication of collaborative trial, magnesium sulphate must be regarded as the agent of choice as it controls eclamptic convulsions and prevents recurrence of convulsions more effectively than diazepam and phenytoin.

In India especially in rural areas, the majority of eclampsia cases report to hospital in an emergency. They are unregistered with inadequate antenatal care and travel a long distance before reaching the hospital as the rural health centres are not sufficiently equipped to manage this condition, so the patients are referred to tertiary care centres.

Though eclampsia is a common disorder few obstetricians have extensive experience of its management. A wide variety of magnesium sulphate protocols have been used in treating eclampsia. Pritchard J.A. ${ }^{5}$ (1955) gets the credit of popularizing magnesium sulphate therapy for eclampsia and preeclampsia in modern obstetrics.

Flower et al. ${ }^{6}$ adjusted doses of magnesium sulphate according to body weight, plasma levels and urinary excretion of magnesium sulphate. Pritchard J.A. commented that the dose of magnesium sulphate should be limited in women who are known or appear to be small (low weight), administrating Pritchard regime might prove to be hazardous with a possibility of respiratory failure.

Indian women weigh much less than their counterparts in the western world, with an average weight of Indian women being 40-45 Kgs, the dose in Pritchard regime is likely to give rise to toxicity.

The purpose of this study was to evaluate the efficacy of low dose magnesium sulphate regime with Pritchard regime and also to evaluate the maternal and perinatal outcome.

\section{AIMS AND OBJECTIVES}

1) To determine the efficacy of low dose magnesium sulphate in eclampsia and imminent eclampsia.

2) To measure serum levels of magnesium with this regime.

3) To correlate serum magnesium levels and efficacy.

4) To identify complications of magnesium sulphate therapy in the mother and the baby.

\section{MATERIAL AND METHODS}

This prospective study was done over two years in the Department of Obstetrics and Gynecology, KIMS, Karad. A pilot study was carried out initially with low dose magnesium sulphate in ten cases of eclampsia after applying the following exclusion criteria.

a) Patients with a history of any other anticonvulsant treatment from the referral hospital before admission.

b) Patients presenting with complications like cerebrovascular accidents, renal failure and aspiration pneumonitis.

\section{Loading dose}

Four grams magnesium sulphate (diluted in normal saline as $20 \%$ solution) was given intravenously over 4 to 5 minutes.

\section{Maintenance dose}

Two grams (50\%) magnesium sulphate intramuscularly was given every three hourly till 24 hours after delivery or last convulsion whichever was later. If the patient got convulsions 20 minutes after the loading dose or anytime after that, while the patient was on maintenance dose then the patient was shifted to a different regime. Also, serum magnesium levels were done. $1^{\text {st }}$ sample at 30 minutes after loading dose and $2^{\text {nd }}$ at 3 hours after $1^{\text {st }}$ dose in all patients. Low dose magnesium sulphate regime was different from Pritchard regime in the following way.

\section{Control of hypertension}

Calcium channel blocker nifedipine was used orally or sublingually for control of hypertension.

\section{Maintenance of fluid and acid-base balance:}

Hydration marinated by ringer lactate solution $1000 \mathrm{cc}$ over 24 hours titrated in response to the urine output. The patient was encouraged to take fluids orally as soon as they recovered consciousness.

\section{Obstetric management}

The decision of termination of pregnancy in all cases of eclampsia and imminent eclampsia was taken.

All patients were monitored for evidence of magnesium toxicity in the form of absence of deep tender reflexes and decreased urine output, depressed respiration, before every dose and if any found, the next dose was withheld.

During every delivery, the pediatrician was available to manage the newborn. Subsequent neonatal care was given by paediatrician till discharge from hospital. Relevant information of every case was entered in the proforma and master chart and final analysis of the important data was done with the help of a statistician.

\section{OBSERVATIONS AND RESULTS}

The maximum number of patients were between the age group of 20-25 years (76.67\%). Incidence of eclampsia was 
more common in primigravida $(61.67 \%)$. A maximum number of patients had weight less than $50 \mathrm{kgs}(74 \%)$. The average weight of all the patients was less than $50 \mathrm{kgs}$.

Maximum patients were between 32-37 weeks of gestation (56.8\%). A maximum number of patients had less than 3 convulsions in the study. The dose required to control convulsion in low dose magnesium sulphate regime is $54.5 \%$ less than the standard Pritchard regime. By applying the Chisquare test it is statistically significant. $(p<0.05)$

Six patients in low dose regime and 5 patients in the Pritchard regime had a recurrence of convulsions after magnesium sulphate.

By applying the Chi-square test it is statistically insignificant.

The success rate in case of imminent eclampsia was $100 \%$ in both the groups.

The success rate in cases of eclampsia was $84.62 \%$ in the low dose group while $87.18 \%$ in Pritchard group.

Applying T-test it was found that there is a significant difference $(p<0.001)$ in serum magnesium levels range in both groups but no statistical difference in the control of convulsions in both groups.

In all the patients the cost of therapy was below 300 Rs in low dose group while it was more than 300 Rs in Pritchard group in $90 \%$ of patients.

LSCS was the most common mode of delivery.

Perinatal mortality was $30 \%$ in low dose regime group and $41.67 \%$ in Pritchard group.

\section{DISCUSSION}

In the present study, it was observed that $90 \%$ of the cases were unbooked or unregistered cases and presented to the hospital with convulsions. Helmin ${ }^{7}$ in 1952 stated that eclampsia will be a clinical rarity if effective antenatal care is made available. As per WHO criteria pregnant women should have at least four antenatal visits throughout the pregnancy, 2 of them preferably in the third trimester (Safe Motherhood Programme, Ratnam 1992.)

From the data collected from this study incidence of eclampsia was high in the age group up to 25 years i.e. $85 \%$. Lehmann in 1933 showed that girls in their early teens and women aged 35 years and more were at increased risk. This may be due to increased awareness of education in women and the raised age for marriage. The incidence of eclampsia is more in this age group as this is the period coinciding with the first conception.

A study by Yadav \& Nayak ${ }^{8}$ quoted age incidence of $83.3 \%$ below the age of 25 years. Lopez and Llera ${ }^{9}$ (1967). Dutta
(1978) and Biswas showed a similar incidence of eclampsia in below 25 years age group i.e. $90.8 \%, 67.3 \%$ and $80 \%$ respectively. In the present study, the incidence of eclampsia below 25 years was $85 \%$.

In the present study, a higher incidence of eclampsia was found in primigravida i.e. $61 \%$ while in multigravida it was $39 \%$. Pritchard (1984) in his study observed that the incidence of eclampsia in primigravida was $75 \%$. As it is thought that severe pre-eclampsia and eclampsia have a familial tendency, and though it is mostly confined to primigravida, their prevalence in multigravida especially due to changing paternal influence is well documented (Nelson 1955 and Chin ${ }^{10}$ 1982). Mudaliar and Menon ${ }^{11}$ (1972) and Dawn ${ }^{12}$ in (1974) reported a $75 \%$ incidence of eclampsia in primigravida.

It is observed that the Indian women, especially staying in rural areas and from middle and lower socio-economic class weigh much below than women from high socio-economic class.

In the present study, it was observed that $74 \%$ of the pregnant women had body weight less than $50 \mathrm{kgs}$. J A Pritchard ${ }^{5}$ himself suggested that "If a woman is known to be or appear to be small the dose should probably be limited." Winit Phuapradit ${ }^{13}$ suggested that it seems appropriate to take into account body weight when considering the dosage of drug and regime used is appropriate for Asian women with bodyweight usually less than $70 \mathrm{Kg}$. In recent years Andrea Witlin ${ }^{14}$ suggested that one may also speculate that magnesium sulphate dosing should vary according to the patient's weights or body mass index. However, this has never been evaluated. These above comments give ample evidence that the dose regime needs to be modified according to patients weight. The low dose of magnesium sulphate regime which has been proposed in this study, the result confirms that this is an adequate dose for Indian women.

In Kameshwari Devi series (1976) out of 363 patients, $69 \%$ were between 32-36 weeks of gestation. In the present study, $56.8 \%$ cases were between $32-37$ weeks gestation and $23.8 \%$ had gestational age more than 37 weeks.

The very purpose of the present study was to find whether a lower dose of magnesium sulphate can control \& prevent convulsions or not and while doing so to measure the serum magnesium levels that are obtained.

Patients on standard Pritchard's regime receive 44 grams of magnesium sulphate in 24 hours from admission. In the present study, we observed that $90 \%$ of patients got control of convulsions with less than 20 grams of magnesium sulphate i.e. $54.5 \%$ less than standard Pritchard regime.

Analysis of the 6 cases of eclampsia which did not respond to an initial loading dose of 4 grams (low dose) of $20 \%$ magnesium sulphate I.V. showed that in 5 of the 6 patients more 
number of convulsions $(>3)$ before initiation of anticonvulsant therapy as compared to cases with less number of convulsions. The cases with more number of convulsions have greater derangement (metabolic and cerebral) than those with lesser number of convulsions. Lower doses of magnesium sulphate through low dose regime could be insufficient to control these gross derangements leading to recurrence of convulsions. Also with Pritchard regime, there were 5 cases of eclampsia patients, those had recurrences after the loading dose or while on maintenance dose indicating that low dose was as effective as Pritchard in control of convulsions.

\section{CONCLUSION}

Low dose magnesium sulphate regime was effective in seizure prophylaxis in imminent eclampsia patients and as effective as Pritchard regime in eclampsia patients. The dose required for control of convulsions was less than half used in Pritchard regime and even cost of therapy was half than Pritchard regime.

Acknowledgement: Authors acknowledge the immense help received from the scholars whose articles are cited and included in references to this manuscript. The authors are also grateful to authors/editors/publishers of all those articles, journals and books from where the literature for this article has been reviewed and discussed.

Conflict of interest: Nothing to report.

Source of funding: Kimsdu Karad

\section{REFERENCES}

1. Chesley LC. A short history of eclampsia. Obst and Gynec 1974; 43: 599 .

2. Williams: Williams obstetrics 22nd International edition, 2005; 761-808.

3. The eclampsia trial collaborative group. Which anticonvulsant for women with eclampsia? Evidence from the collaborative eclampsia trial. The Lancet, 1995; 345:445-63.

4. Magpie trial collaborative group: Do women with preeclampsia and their babies benefit from magnesium sulphate? The Magpie Trial: A randomized placebo-controlled trial. Lancet, 2002; 359: 1877.

5. Pritchard JA, Cunningham FG, Pritchard SA: The Parkland Memorial Hospital protocol for the treatment of eclampsia: Evaluation of 245 cases. Am J Obstet Gynecol 148: 951, 1984.

6. Flower CA: Magnesium sulphate in toxaemia of pregnancy-new dosage schedule based on body weight. J Obstet Gynecol; 1962; 19:315-318.

7. Helmin RH J, Prevention of Eclampsia and preeclampsia; Lancet $1980 ; 64$.

8. Yadav and Nayak; Eclampsia-A report of 54 cases. Jr. of Obstet and Gynecol of India. 1980; 30:902.

9. Mario Lopez Llera: Complicated eclampsia. Am J Obstet Gynecol. 1982; 142L:28-35.

10. Chin Chu Lin: Fetal outcome in hypertensive disorders of pregnancy. Am J Obstet Gynecol 42:256-260, 1982.

11. Mudaliar \& Menon MKK: Clinical Obstetrics. 7th Ed. 1971.

12. Dawn CS: Textbook of Obstetrics and Gynecology. 1974.

13. Phaupradit Winit, Saropala N et al: Serum level of magnesium attained in magnesium sulphate therapy for severe preeclampsia. Asia Oceania J Obstet Gynecol. 1993;194:387-390.

14. Witlin Andrea: Prevention and Treatment of eclamptic convulsions. Clinical Obstet and Gynecol. 1979;42(3): 126-130. 